\title{
Research on an intelligent traffic congestion control model
}

\author{
Bo Xu, Jianbing Chen*, and Wei Tang \\ Computer Science, Network and Information Center, 650500 Yunnan Normal University, China
}

\begin{abstract}
This paper summarizes the status quo of intelligent traffic congestion control and vehicle following on traffic road, puts forward the key technology model and its content of intelligent traffic control, elaborates the model and content in detail, and summarizes the research done, hoping to provide reference for the related research on intelligent traffic congestion control.
\end{abstract}

\section{Introduction}

In October 18, 2017, general secretary Xi Jinping made clear for the first time in the party's nineteen major reports the development strategy of building a "transportation power" [1]. On May 31, 2019, the transportation informatization standard system (2019) ${ }^{[2]}$ proposed to promote the innovative application and development of cloud computing, big data, Internet of things, mobile Internet, artificial intelligence and other information technologies in the transportation industry.

On July 25, 2019, the Ministry of transport issued the outline of digital transportation development planning ${ }^{[3]}$, proposing that the development of digital transportation should take "data chain" as the main line, build a digital collection system, a networked transmission system and an intelligent application system, accelerate the development of transportation information to digital, networked and intelligent, and provide support for the construction of a transportation power The content is to build a high-precision transportation geographic information platform covering the whole country, improve the information of traffic engineering and other elements; lay out the omni-directional traffic perception network of important nodes, establish the perception network of cloud interconnection, so that the "dumb facilities" have the ability of multi-dimensional monitoring, intelligent network connection, precise management and control, and collaborative service; and encourage those with multi-dimensional perception, high-precision positioning and intelligent network connection functions The application of terminal equipment promotes the research and development of automatic driving and vehicle road collaborative technology.

On September 19, 2019, the CPC Central Committee and the State Council officially issued the outline for the construction of a transportation power ${ }^{[4]}$, which calls for the vigorous development of smart transportation: promoting the deep integration of big data, Internet, supercomputing and other new technologies with the transportation industry;

\footnotetext{
${ }^{*}$ Corresponding author: jackiesir@ynnu.edu.cn.
} 
promoting data resources to enable the development of transportation, accelerating the integration and development of transportation infrastructure network and information network, and building ubiquitous advanced transportation information infrastructure Build a comprehensive transportation big data center system.

\section{Research status and development trends at home and abroad}

\subsection{Research status of intelligent traffic congestion control}

According to literature ${ }^{[5]}$, traffic congestion presents a radiating conduction characteristic in the road network, and the congested sections tend to diffuse the congestion to other adjacent sections, and the congestion modeling is conducted from the perspective of time and big data rule mining; the time series rule mining algorithm is used to build the interchange congestion conduction law model, and the future traffic flow situation is predicted based on the conduction rules.

It is of great significance to analyze traffic flow through scientific prediction algorithm for congestion control research. Linear model prediction methods for short-term traffic flow analysis mainly include historical average method (HA), exponential smoothing method, time series method (based on AR model, MA model, ARMA model, ARIMA model, etc.), Kalman filter, etc.; nonlinear models mainly include principle model, empirical model and mixed nonlinear model ${ }^{[6,7]}$. The autoregressive analysis model based on time series has been widely used in traffic flow prediction and decision-making ${ }^{[8]}$, including a large number of data smoothing and average data process, loss of certain time accuracy, thus affecting its prediction performance. Linglas and osbaum found that regression analysis and neural network model have good adaptability to dynamic environment ${ }^{[9]}$. At the same time, as the basic map services with low accuracy in the market, the research on traffic congestion prediction and conduction law is essentially the same, and has always been lagging behind, which can not avoid the occurrence of "secondary congestion".

Zhang Shuai ${ }^{[10]}$ thinks that vehicle lane changing behavior is one of the most basic driving behaviors under expressway conditions, and it is also the main cause of traffic congestion and accidents. At present, most of the lane change decision models ${ }^{[11]-[13]}$ are based on microscopic traffic system design, and consider the individual behavior of a single vehicle, such as Audi ${ }^{[14]}, \mathrm{BMW}^{[15]}$, Ford ${ }^{[16]}, \mathrm{GM}^{[17]}$, benz ${ }^{[18]}$, and Mazi The lane change assistant system such as $\mathrm{Da}^{[19]}$ can remind the driver whether there is an approaching vehicle in the adjacent lane, so as to reduce the probability of collision between the vehicle and the vehicle in the adjacent lane during lane change. However, the effect in the actual traffic environment is not very ideal, and there is no scientific and reasonable judgment method for lane change safety.

\section{2 research status of vehicle following on traffic road}

The concept of car following was first proposed by reuschel et al. The model assumes that the driver keeps an expected relative distance from the vehicle in front by controlling the speed of the vehicle, and assumes that the expected distance is linearly related to the speed of the following vehicle ${ }^{[20,21]}$. The research on car following model is of great significance to traffic congestion prevention. For example, literature ${ }^{[20]}$ studied the road traffic scene type identification, traffic scene segmentation, lane line identification as the basis, and selected the characteristic parameters which can well represent the driving risk such as free flow state, car following state and lane change state, and designed the free flow state based on fuzzy reasoning The membership function of characteristic parameters and driving risk 
reasoning rules under car following state and lane changing state. In reference ${ }^{[22]}$, an improved safety distance car following model based on optimal speed model was proposed to overcome the defect of constant safety distance assumption in the optimal speed (OV) model and its related derivative models (GF, FVD, etc.).

At the same time, reference ${ }^{[23]}$ considers that the mainstream traffic data used in the calibration and validation of car following model is the next generation simulation, Ngsim) data based vehicle trajectory data. These high-resolution trajectory data only record the kinematic characteristics of the vehicle. The amount of data used for parameter calibration is small and the acquisition cost is high. The future data comes from various sensors, which has the advantages of accuracy, full time and no sampling error. Big data can be used to establish the car following model of the optimal solution and design the algorithm In order to improve the automation of the calibration process and enhance the applicability of the model, the optimal solution is calculated.

\section{Key technologies of intelligent transportation governance model}

\subsection{Related contents}

(1) Dynamic tree of vehicle (or group of vehicles)

(1) The edge between the vehicles in front of and behind the multi branch tree is a real edge, while the edge between vehicles in different lane relationships (left front, right rear) is virtual;

(2) In the process of driving, multiple local vehicle multi tree gradually gather together to form a larger multi tree;

(3) Root searching and anti congestion: assuming that the global or local root is the key node for traffic congestion, the process of traffic congestion prevention is to find the root of the tree. Finding the node that is most likely to form traffic congestion can complete the prevention of traffic congestion on the road.

(4) Gateway: when vehicles enter the gate and become the corresponding branch of a tree, a branch of the dynamic tree needs to be subtracted or cut off for the vehicles driving out of the gate. When the middle node of a branch of a dynamic tree drives out of the gateway, it is necessary to cut off the node here and re connect the new branch; if the end node of the branch leaves the gateway, the node can be cut off directly;

(5) Because the vehicles on the expressway are in motion, except for overtaking and lane changing, assuming that the driving speed of the detection section is relatively uniform and relatively static, this paper carries out the expansion based on this ideal state.

(2) Dynamic tree based resource management

Dynamic tree is a description of the driving state relationship between vehicles within a certain range (within $100 \mathrm{~km}$ ). Here is a dynamic relationship between all vehicles that will affect the current vehicle running and within a certain range before and after the current vehicle driving.

(1) Sum on Chain: find the length and longitudinal density of a branch of the tree. Knowing this value, you can recommend the best driving lane for the following vehicles.

(2) Modification on the chain: variables such as speed, lane and position are constantly changing with the vehicle;

(3) Subtree modification;

(4) Change root: on the dynamic tree, the top one is the root; 
(5) Break an edge off the tree. When one or more vehicles no longer belong to a part of the dynamic tree, the branch should be disconnected or cut off from the dynamic tree.

(3) Multi branch lane changing equilibrium tree

In order to better overtake on expressways, the vehicles behind will always drive to the lane with the least number of vehicles. After numerous "catch-up" changes (deformations), a relatively stable shape will be formed, which can be described by several changes in the following figure. We call the following situation "greedy" driving, as shown in Figure 1.

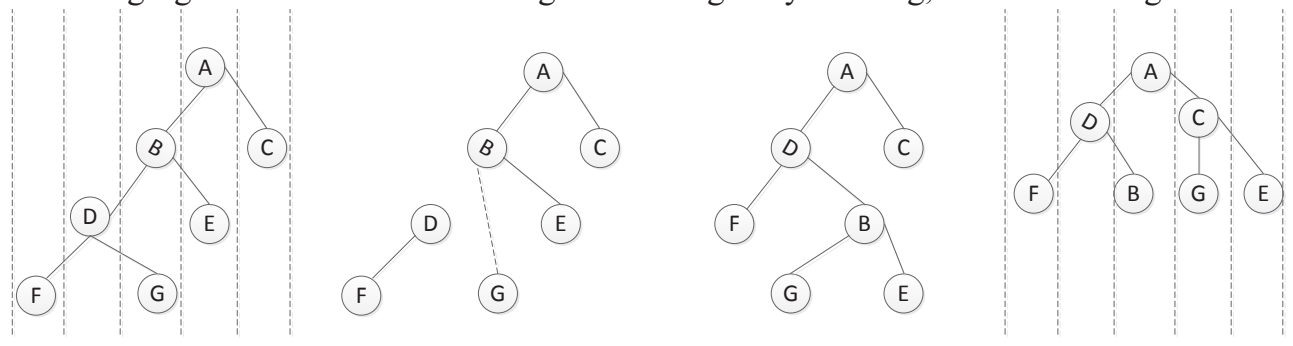

Fig.1. Multi Lane driving balance tree.

(4) Stretch tree, change lane, overtake and follow

Similarly, there is a overtaking process when the vehicle is driving on the expressway, which can be described by several changes in Figure 2.

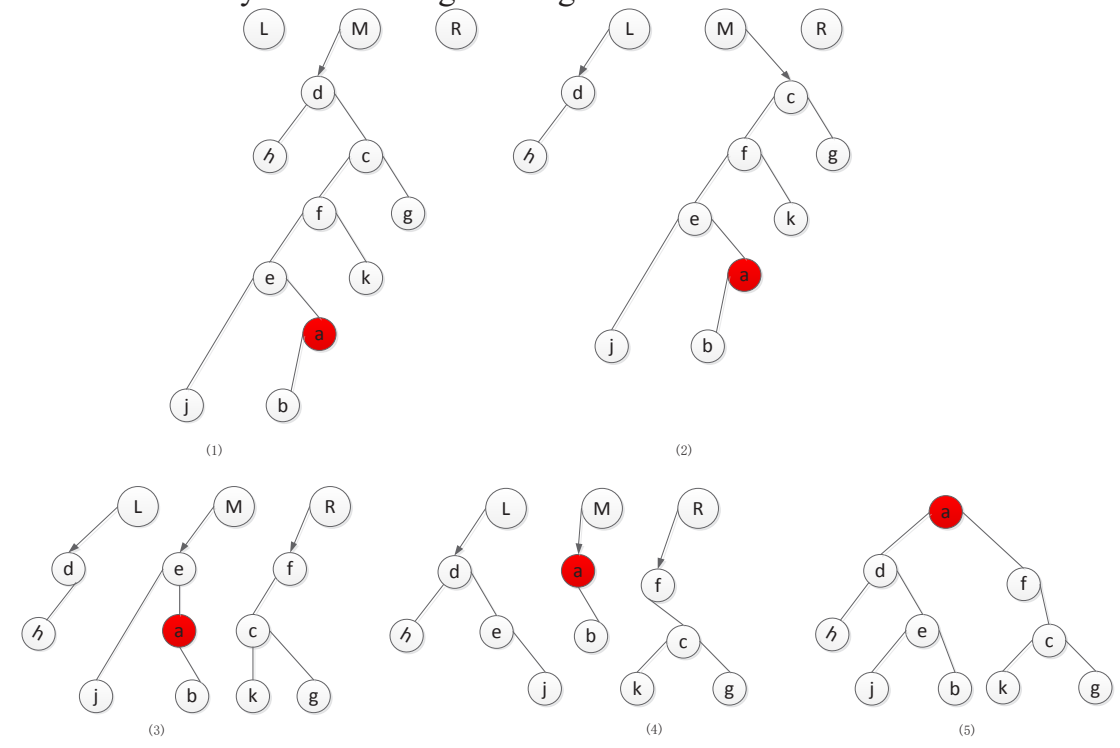

Fig. 2. following overtaking.

On the existing traffic road experimental data analysis, or experimental simulation of the upper stretch tree overtaking process.

\subsection{Local control technology of lane line (dotted line and solid line)}

According to the use of road resources, the virtual and real working state of local or global road lane is dynamically controlled by vehicle driving tree model, so as to guide traffic vehicles to go their own way in the best way. Traffic congestion prevention is the result of the interaction between road elements such as lane lines and lanes and numerous dynamic vehicles. From the perspective of each local road, the algorithm is fully utilized to control 
the road elements and dynamic vehicle driving, so as to realize the interaction and cooperation between the road, vehicles and vehicle groups, so as to achieve a safe and reliable traffic road The purpose of parallel use of resources.

(1) Parallel control of multi Lane vehicles

Due to the existence of multi lanes, multi vehicles use road resources in parallel, or in time or order or priority (such as overtaking and following); the use of resources will inevitably lead to competition (vehicle head collision) or improper sequence (rear end), so it is necessary to rationalize the use of road resources.

(2) Algorithm of shortest time occupation of road by vehicle group

It is a way to prevent traffic congestion by completing the driving of multiple vehicles on multiple lanes in the shortest time. Multi vehicle is regarded as multiple operations, multi lane is regarded as multiple machines, and multi vehicle driving on multi lane is regarded as multi operation, which needs to be processed by multiple processors. Each vehicle completes the driving on different lanes through lane changing, overtaking and following, and finally completes the local road driving; according to the driving situation of each vehicle, through the shortest time occupation Lane algorithm, for each "overtaking and following" group of vehicles, it dynamically calculates that each section of road needs to control the overtaking and following conditions to control the virtual and real changes of lane line, and guide the shortest time of vehicle group Use vehicles to avoid traffic congestion in local vehicle groups.

(3) Optimal speed recommendation based on safe following distance

On the premise that the road is virtualized and accurately managed through the Internet of things, it is proposed to integrate the vehicle control technology based on the safe car following model ${ }^{[24]}$ (the following process) into the driving process of the two control technologies mentioned above, so as to achieve the best driving speed recommendation of vehicles. The first is the acceleration (deceleration) model

$$
a_{\text {follower }}=\left\{\begin{array}{l}
\left(\frac{V_{o v}-V_{\text {follower }}}{V_{f \text { max }}-V_{\text {follower }}}\right)^{a} \bullet A_{\text {facc max }}, \quad \mathrm{x}_{\text {gap }} \geq \mathrm{D}_{\text {safe }} \mathrm{andV}_{\text {follwer }}<V_{f \text { max }} \\
\left(\frac{V_{\text {follower }}-V_{o v}}{V_{\text {follower }}-V_{f \min r}}\right)^{a} \bullet D_{\text {facc max }}, \quad \mathrm{x}_{\text {gap }}<\mathrm{D}_{\text {safe }} \text { and } V_{\text {follwer }}>V_{f \text { max }}, \quad \mathrm{x}_{\text {gap }} \geq \mathrm{D}_{\text {safe }} \text { and } V_{\text {follwer }}=V_{f \text { max }}
\end{array}\right.
$$

where: ${ }^{a_{\text {follower }}}$ is the acceleration of the current driving behavior of the following vehicle; $\mathrm{D}_{\text {faccmax }} \leq a_{\text {follower }} \leq \mathrm{A}_{\text {faccmax }}$ is the optimal speed of the following vehicle without collision; $V_{\text {fmax }}$ is the maximum driving speed of the road following vehicle; $V_{\text {fmin }}$ is the minimum driving speed of the road following vehicle (the value $V_{\text {fmin }}$ is $0 \mathrm{M} / \mathrm{s}$ ); $\mathrm{V}_{\text {follower is the current driving speed of the following vehicle; }} \mathrm{V}_{\text {faccmax }}$ is the maximum acceleration of the following vehicle; $\mathrm{D}_{\text {faccmax }}$ is the maximum deceleration of the following vehicle; $\mathrm{X}_{\text {gap }}$ is the centroid distance between the following vehicle and the vehicle ahead; $a$ is the correction coefficient.

The optimal speed model is defined as follows: 


$$
\mathrm{V}_{\text {ov }}=\left\{\begin{array}{l}
\left(\mathrm{V}_{\text {follower }}-\mathrm{V}_{\text {fmin }}\right) \bullet \tanh \left(\left(\frac{\mathrm{x}_{\text {gap }}-\mathrm{D}_{\text {safe }}}{\mathrm{D}_{\text {safe }}-\mathrm{D}_{\text {minsafe }}}\right) \bullet \mathrm{b}\right)+ \\
\mathrm{V}_{\text {follower }}, \quad \mathrm{x}_{\text {gap }}<\mathrm{D}_{\text {safe }} \text { and } \mathrm{V}_{\text {follower }}>\mathrm{V}_{\text {fmin }} \\
\left(\mathrm{V}_{\text {fmax }}-\mathrm{V}_{\text {follower }}\right) \bullet \tanh \left(\left(\frac{\mathrm{x}_{\text {gap }}-\mathrm{D}_{\text {safe }}}{\mathrm{D}_{\text {maxsafe }}-\mathrm{D}_{\text {safe }}}\right) \bullet \mathrm{b}\right)+ \\
\mathrm{V}_{\text {follower' }}, \mathrm{x}_{\text {gap }} \geq \mathrm{D}_{\text {safe }} \text { and } \mathrm{V}_{\text {follower }}<\mathrm{V}_{\text {fmax }} \\
\mathrm{V}_{\text {fmax }}, \mathrm{x}_{\text {gap }} \geq \mathrm{D}_{\text {safe }} \text { and } \mathrm{V}_{\text {follower }}=\mathrm{V}_{\text {fmax }}
\end{array}\right.
$$

where: $\mathrm{D}_{\text {minsafe }}$ is the safe distance between the center of mass of the following vehicle and the vehicle in front of it when it stops; $\mathrm{D}_{\text {maxsafe }}$ is the safe distance between the center of mass of the car following it when it is running at the maximum speed; $\mathrm{D}_{\text {safe }}$ is the safe distance of the center of mass between the following vehicle and the vehicle in front when it is running at the current speed; B is the correction coefficient.

Through the interaction among the acceleration, speed and distance of the following vehicles, the safe non rear end control of vehicles is realized, and the effect of multi local car groups following on the global road following on the same traffic road (butterfly effect) and its congestion control are further studied, so as to provide scientific basis for the dynamic speed limit value of different sections. Based on this, the precise big data environment is studied Car following model.

\subsection{Road management based on accurate big data prediction}

Through the multi-source sensor model, we can fully and completely collect and process the basic data such as global or local road resources and vehicle driving conditions, and build a traffic road resource big data management center. Based on big data technology, this paper studies the integrated scheduling algorithm of road resources and deep learning, load balancing, anti deadlock, etc., predicts the pre governance area of vehicle free traffic congestion and its early deployment and management: preprocesses and initializes the traffic lights, lane lines, and the optimal speed limit value of speed limit area on the road resources to be used globally or locally, and guides the vehicles or vehicle groups in advance The occupation and recovery of multi vehicle road resources in this area.

Finally, the above dynamic control algorithm will be applied to the traffic road elements under dynamic control. The basic model is shown in Figure 3 below. 


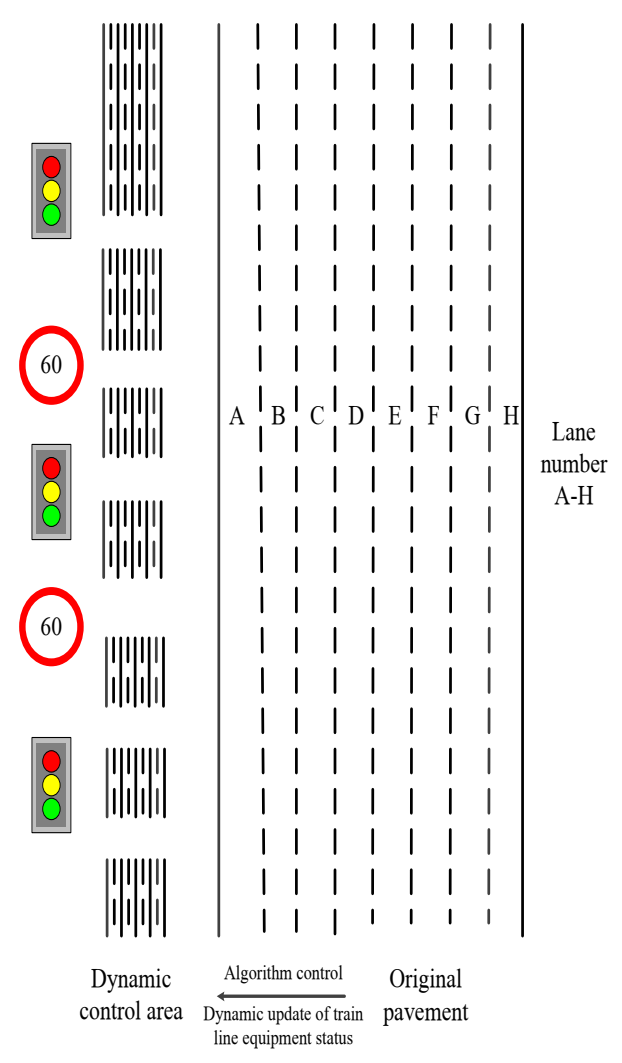

Fig. 3. Dynamic control model of lane.

According to the accurate traffic resources and control algorithm after the virtualization of the Internet of things, the dynamic control and governance of the basic elements of local and global traffic roads, such as traffic lights, lane lines and speed limit areas, are realized.

\section{Summary}

This paper studies the scheduling algorithm of time variable, road resources, vehicles and other basic elements to work together. On the basis of accurate management of traffic road resources, the algorithm can be used to guide the rational and scientific use of road resources from local to regional, from regional to overall through the dynamic scheduling of algorithm on the road variable factors such as lane line, traffic light and maximum speed It can accurately control traffic congestion. Based on the virtual calculation of traffic road resources and its big data platform data management, the use of resource consumption dynamic scheduling algorithm, real-time monitoring and deployment of traffic lane lines, traffic lights, speed limit areas and other elements, to guide the orderly, efficient and smooth use of traffic road resources.

Supported by the science research fund of Yunnan Education Department in 2019

\section{References}

1. The 18th Central Committee of the Communist Party of China. A decisive victory in building a moderately prosperous society in all respects and winning the great victory 
of socialism with Chinese characteristics in the new era [R]. Beijing: the 18th Central Committee of the Communist Party of China, 2017

2. Ministry of transport of the people's Republic of China. Notice on the issue of transport information standard system (2019) (Jiaoban science and technology [2019] No. 49) [Z]. Beijing: Ministry of transport of the people's Republic of China, 2019

3. Ministry of transport of the people's Republic of China. Notice on printing and distributing the outline of digital transportation development plan (jgjf [2019] No. 89) [Z]. Beijing: Ministry of transport of the people's Republic of China, 2019

4. Central Committee of the Communist Party of China, State Council of the people's Republic of China. Outline of building a transportation power [Z]. Beijing: Central Committee of the Communist Party of China, State Council of the people's Republic of China, 2019

5. Zhou Huiyu. Research on traffic congestion management based on big data rule mining [J]. Forum on statistics and information, 2017,32 (05): 96-101

6. Hsu C W, Lin C J.A Comparison of Methods for Multiclass Support Vector Machines[J].IEEE Transactions on Neural Networks, 2002 (2) .

7. Bock M A, Bowman W, Ismail B.Estimation and Inference for Error Variance in Bivariate Nonparametric Regression[J].Statistics and Computing, 2007 (1) .

8. Yoon Byoungjo, Chang H.Potentialities of Data-Driven Nonparametric Regression in Urban Signalized Traffic Flow Forecasting[J].Journal of Transportation Engineering, 2014 (7) .

9. Lingras P J, Osborne P.Effect of Noise on Regression and Neural Network Predictions[C].In Proc.of the Conference of Canadian Society of Civil Engineers, Sherbrooke, Quebec, 1997.

10. Zhang Shuai. Research on autonomous lane changing algorithm of intelligent vehicle under expressway [D]. Jilin University, 2019

11. Wang Zheng. Research on autonomous lane changing method of intelligent vehicle [D]. Jilin University, 2016

12. Esmaeil Balal,Ruey Long Cheu,Thompson Sarkodie-Gyan. A binary decision model for discretionary lane changing move based on fuzzy inference system[J].Transportation Research Part C.2016.

13. M. Rickert,K. Nagel,M. Schreckenberg,A. Latour. Physica A.Two lane traffic simulations using cellular automata[J].Statistical Mechanics and its Applications.1996(4)

14. Popken M. Audi side assist[J]. Hanser Automotive electronics + systems, 2006, 7(7 8):54-56.

15. 7-Series Forum (2008) Press photos published by BMW of the new 7-Series. http://www.7-forum.com/modelle/f01/galerie_730d_750li.php.Accessed 25 May 2010.

16. Ford press release on its Blind spot information system (2009). http://media.ford.com/images/10031/BLIS.pdf. Accessed 25 May 2010.

17. GM homepage (2010) Side blind zone alert in Buick LaCrosse can help avoid lane change

mishaps.http://www.gm.com/corporate/responsibility/safety/news/2010/blind_spot_04 0910.jsp.Accessed 25 May 2010.

18. on Heise Online (2007) Mercedes: new blind spot Assistant. http://www.heise.de/autos/Sund-CL-Klasse-Neuer-Totwinkel-Assistent-fuermehr Sicherheit-beim-Spurwechsel-/artikel/s/4517.Accessed 11 Sept 2008 
19. Article

inAutobild.de(2008).http://www.autobild.de/artikel/mazda6-2.2-mzr-cd_806923.html. Accessed 25 May 2010 orado "Smart Highway" Project. Integrated Roadways. http://integratedroadways.com.2018

20. REUSCHEL A.Vehicle movements in the column uniformly accelerated or delayed[J]. Oesterreich IngrArch,1950,4:193-215.

21. PIPES L A.An operational analysis of traffic dynamics[J].Journal of Applied Physics, 1953,24(3):274-281.

22. Qu Zhaowei, pan zhaotian, Chen Yongheng, Tao Pengfei, sun di. Improved safety distance car following model based on optimal speed model [J]. Journal of Jilin University (Engineering Edition), 2019, 49 (04): 1092-1099

23. Yang Longhai, Zhang Chun, Qiu Xiaofu, Li Shuai, Wang Hui. Research progress of vehicle following model [J]. Journal of transportation engineering, 2019, 19 (05): 125-138

24. Qu Zhaowei, pan zhaotian, Chen Yongheng, Tao Pengfei, sun di. Improved safety distance car following model based on optimal speed model [J]. Journal of Jilin University (Engineering Edition), 2019, 49 (04): 1092-1099 